\title{
Finite element analysis of out-of-plane compressive properties of a honeycomb structures with hexagonal topology fabricated by the kirigami technique
}

\author{
Belloufi Abderrahim, Mustapha Bouakba*, Mourad Boukhatem and Brahim Issasfa
}

\begin{tabular}{l} 
Department of Mechanical Engineering \\
\hline A R T I C L E I N F O \\
\hline Article history: \\
Received 6 September, 2015 \\
Accepted 12 January 2016 \\
Available online \\
14 January 2016 \\
\hline Keywords: \\
FEA \\
Honeycombs \\
Cellular \\
Flatwise \\
Hexagonal topology
\end{tabular}

\begin{abstract}
Aculty of Applied Sciences, University of KasdiMerbah Ouargla, 30000, Algeria
A B S T R A C T

This work illustrates the manufacturing of the honeycomb hexagonal topology structures by the kirigami technical, and the compressive testing of this specimen. The cellular configuration is simulated using a series of finite element models representing fullscale. The models are benchmarked against experimental results from pure compression tests. Finite element models of the honeycomb assemblies under compressive loading have been developed using nonlinear shell elements from an ANSYS code. Good agreement is observed between numerical nonlinear simulations and the experimental results.
\end{abstract}

\section{Introduction}

Honeycomb out-of-plane compressive properties are of interest for many researchers because they are important for the mechanical performance of sandwich panels, such as local compression and impact resistance. Bouakba et al. (2012) proposed a novel type Voronoi-lattice and studied this honeycomb by FEA on in-plane mechanical properties using the ANSYS code. Many researchers have used FEA (e.g. numerical approaches) to better understand and predict the elastic properties of honeycombs structure (Gibson \& Ashby, 1997; Ren et al., 2008, Lascoup et al., 2010; Gong et al., 2005; Zhu et al., 1997; Zhu et al., 2001; Zhu et al., 2007). Chen et al. (2013) calculated the elastic proprieties of the anti-tetra chiral topology, using a digital image correlation DIC and bench-marked the obtained results with the theoretical and the FEA results. Khan et al. (2012) investigated the inplane and out-of-plane crushing properties of the honeycomb core using a digital image correlation. All these works have been done to understand the capacity of using the honeycombs cells in sandwich panels and to know their mechanical proprieties.

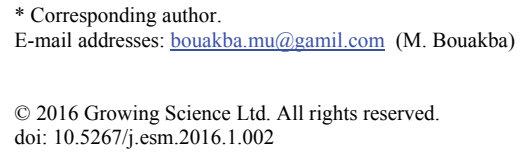


This work describes a study of the out-of-plan mechanical properties of a honeycombs made by krigami technique. The compressive properties for this type of lattice have been simulated using a fullscale representation of the honeycomb panels under compression loading. The experimental evaluations on the hexagonal honeycomb compressive properties are implemented by doing flatwise compressive test (FCT) according to the standard ASTM C365.

\section{Manufacturing and experimental testing}

\subsection{Kirigamihoneycomb structures manufacturing process}

The Kirigami manufacturing process consists in the following three steps: cutting, folding and bonding regular distributions of slits that are introduced within the plain carton paper in the shape of patterns shown in Fig. 1. The cutting lines are generated by MATLAB program based on the algorithm of the hexagonal cell of honeycombs topology with the gauge thickness of the honeycomb walls (Hou et al., 2014; Virk et al., 2013; Saito et al., 2011). The compressive properties of the hexagonal cell topology along the direction parallel to the plane of the sandwich face skin are evaluated through edgewise compression tests according to the ASTM Standard ASTM C364/C364M-07 carried out on a testing machine. The specimen size $(50 \mathrm{~mm} \times 50 \mathrm{~mm} \times 28 \mathrm{~mm})$ satisfies the requirements of the ASTM standard, while being quite similar to the one used to dimension the specimens for flatwise compression tests. The specific machine configuration setup is the same used for the flatwise testing, with the bottom plate fixed and the top plate moving downwards at a constant velocity of $0.5 \mathrm{~mm} / \mathrm{min}$. five specimens are tested in total, and different failure modes are observed during loading.

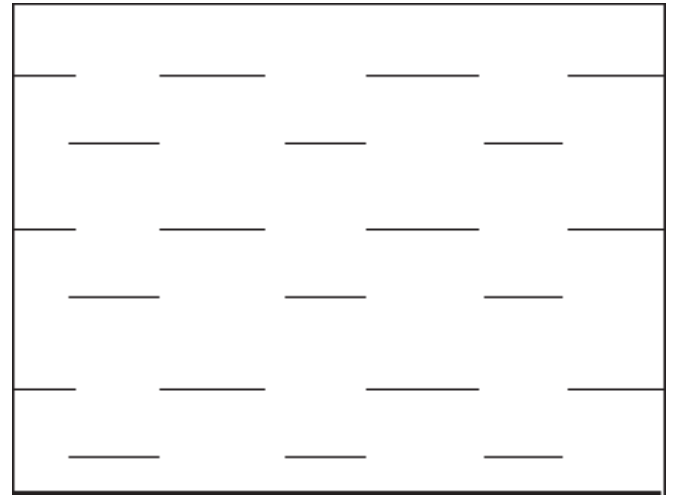

(a)

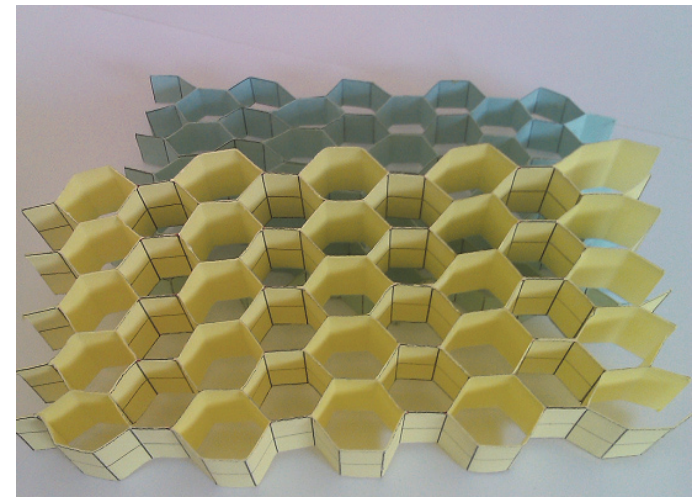

(b)

Fig. 1. a ) cutting lines; b) Paper hexagonal honeycombs obtained by krigami technique.

\section{Finite element models}

Fig. 2 shows the finite element model of honeycomb developed using the commercial code ANSYS 11.0. The out of plane compressive properties have been simulated using a full-scale representation of the honeycomb panels under compression loading (Chen et al., 2013). After a mesh convergence analysis, an average mesh size equal to 1/4 was adopted for the SHELL63 elements (Chen et al., 2013; Scarpa et al., 2000; Bouakba et al., 2012). The honeycomb cell walls are modelled by using four-node three dimensional shell elements, SHELL63, in ANSYS. SHELL63 has both bending and membrane capabilities. Both in-plane and normal loads are permitted. The element has six degrees of freedom at each node: translations in the nodal $\mathrm{x}, \mathrm{y}$, and $\mathrm{z}$ directions and rotations about the nodal $\mathrm{x}, \mathrm{y}$, and $\mathrm{z}$ axes. Stress stiffening and large deflection capabilities are included. A consistent tangent stiffness matrix option is available for use in large deflection analysis. The element thickness and orthotropic elastic properties are the main input data to simulate the real material. The modulus is taken as $1 \mathrm{GPa}$ and the 
Poisson's ratio is set as 0.3. A Newton-Raphson nonlinear solver is used to extract the forcedisplacement curve $\mathrm{F}-\delta$.

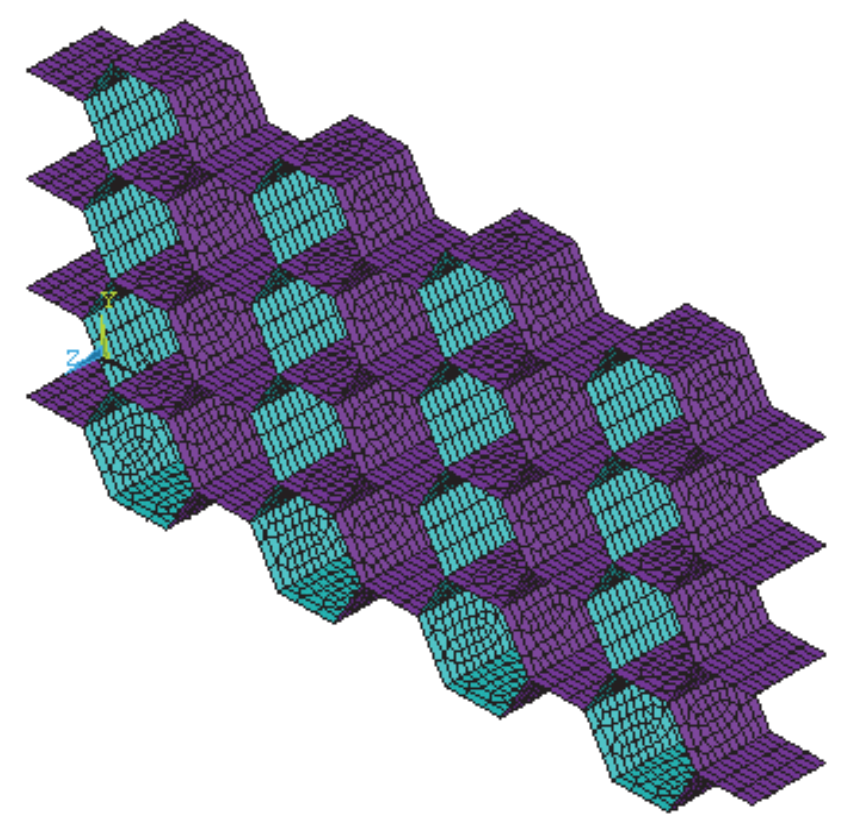

Fig. 2. Full model of honeycombcreated in ANSYS code

\section{Results and discussion}

\section{1. load-displacement behaviour}

Load-displacement curve of the tested honeycombs (as presented in Fig. 3) was used to identify their mechanical characteristics. The compressive deformation process can be categorized into three regions (I, II and III) based on the compressive stress-strain behaviour (Gibson \& Ashby, 1997; Silva et al., 1995). The compressive stress increased almost linearly with increasing strain in region I due to the elastic buckling of cell walls, not the elastic axial shortening of cell walls. The cell walls of honeycomb composite were restrained with the neighbouring cell walls which are very thin. Therefore, when the compressive deformation is performed to the honeycomb, it is more difficult to be happened by the elastic axial shortening of cell walls rather than by the elastic buckling of cell walls. When the stress-strain curve passed a maximum stress, a rapid drop of the compressive stress appeared in region II due to the onset of the plastic buckling of cell walls. The reason of a rapid drop of the compressive stress in region II is that the elastic buckling of cell walls was converted quickly to the plastic buckling of cell walls. The plastic buckling was initiated at the free walls and propagated into the ribbon. Then, the deboning fracture of cell/cell interfaces at ribbon was followed in region II. In plateau region III, the fracture of resin layer on cell walls was proceeded continuously. It is reasonably thought as the plastic shortening that the nearly constant load proceeded in region III with the fracture of resin layer on cell walls. The compressive stress was maintained nearly constant up to a failure in region III. 


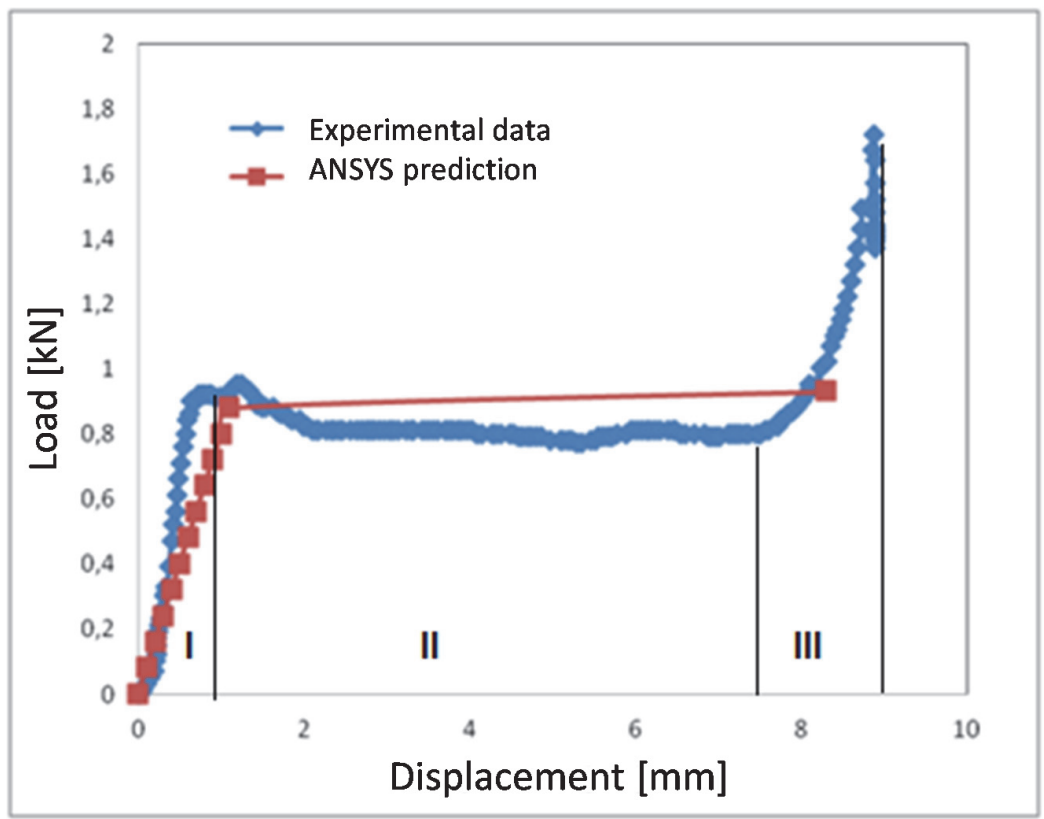

Fig. 3. Load-displacement curve obtained from the experimental compressive tests.

\subsection{Deformation mechanisms}

Deformation of tested honeycomb structure was categorized in three following stages:

- First stage: Appearance of a local buckling of the wall

A local buckling-shaped deformation of the cell wall was gradually appeared on the faces of the hexagon as shown in Fig.4. This buckling occurs in the elastic region of the curve load-displacement (as shown in Fig. 3).

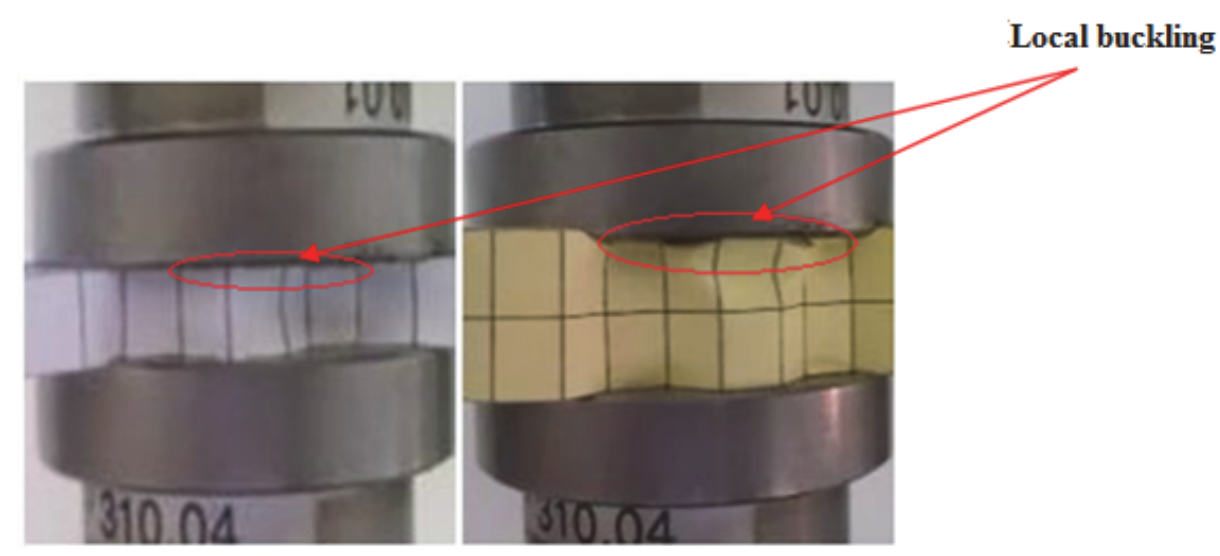

Fig. 4. Local buckling on the hexagonal faces.

- Second stage: appearance of the first damage.

The passage of the critical force (the yield stress), the vertical edge flames locally and begins to be pressed. Simultaneously, the first ply begins to form. It is formed either inside or outside the line of the initial hexagonal cell as shown in Fig. 5. 


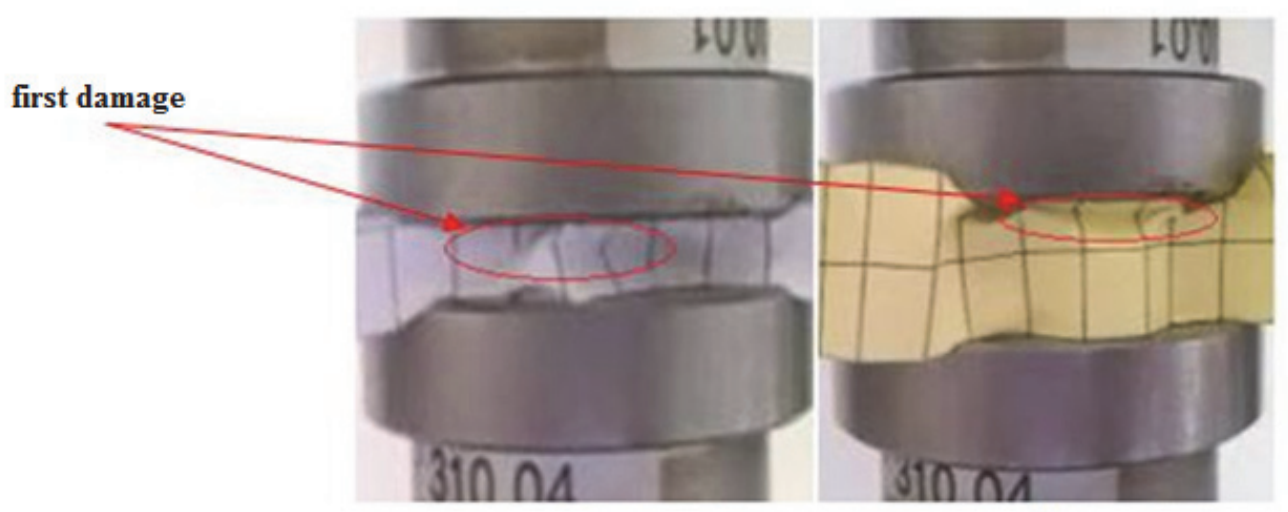

Fig. 5. Formation of the first ply failure.

\section{- Third stage: folding and flattening of the structure}

In this stage, while the deformation of the first ply is flattened completely, the second bend begins to form and the deformation is observed in the form of tear or peel off as shown in Fig. 6.

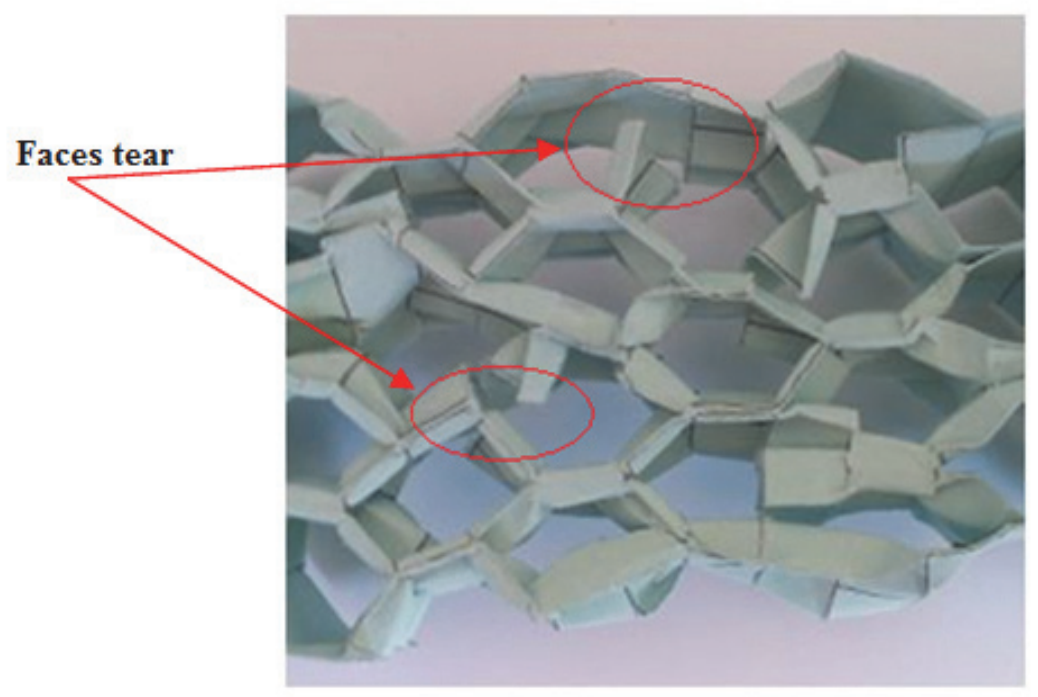

Fig. 6. Hexagonal faces tear of honeycomb structure.

\section{Conclusion}

The main motivation for this study is to propose new alternative cellular cores to be used in the construction of sandwich structures, having geometries that allow the reduction of the base material. As a result, this work presents a Hexagonal topology cellular structure that is proposed to be used as a core in sandwich panels. It aims to develop an FEM model for the out-of-plane effective compressive properties of the proposed cellular structure which is validated through experimental tests. The crushing of the specimen tested was identified the damage scenario. Of particular note, early local buckling of the vertical walls of the cells while the vertical edges remain straight. It is only when they are soaring in turn that the first fold begins to form and the compression force drops suddenly. 


\section{References}

Bouakba, M., Bezazi, A., \& Scarpa, F. (2012). FE analysis of the in-plane mechanical properties of a novel Voronoi-type lattice with positive and negative Poisson's ratio configurations. International Journal of Solids and Structures, 49(18), 2450-2459.

Chen, Y. J., Scarpa, F., Liu, Y. J., \& Leng, J. S. (2013). Elasticity of anti-tetrachiral anisotropic lattices. International Journal of Solids and Structures, 50(6), 996-1004.

Gibson, L. J., \& Ashby, M. F. (1997). Cellular solids: structure and properties. Cambridge university press.

Gong, L., Kyriakides, S., \& Jang, W. Y. (2005). Compressive response of open-cell foams. Part I: Morphology and elastic properties. International Journal of Solids and Structures, 42(5), 13551379.

Hou, Y., Neville, R., Scarpa, F., Remillat, C., Gu, B., \& Ruzzene, M. (2014). Graded conventionalauxetic Kirigami sandwich structures: Flatwise compression and edgewise loading. Composites Part B: Engineering, 59, 33-42.

Khan, M. K., Baig, T., \& Mirza, S. (2012). Experimental investigation of in-plane and out-of-plane crushing of aluminum honeycomb. Materials Science and Engineering: A, 539, 135-142.

Lascoup, B., Aboura, Z., Khellil, K., \& Benzeggagh, M. (2010). Homogenization of the core layer in stitched sandwich structures. Composites Science and Technology, 70(2), 350-355.

Ren, X. J., \& Silberschmidt, V. V. (2008). Numerical modelling of low-density cellular materials. Computational materials science, 43(1), 65-74.

Saito, K., Agnese, F., \& Scarpa, F. (2011). A cellular kirigami morphing wingbox concept. Journal of intelligent material systems and structures, 22(9), 935-944.

Scarpa, F., Panayiotou, P., \& Tomlinson, G. (2000). Numerical and experimental uniaxial loading on in-plane auxetic honeycombs. The Journal of Strain Analysis for Engineering Design, 35(5), 383388.

Silva, M. J., Hayes, W. C., \& Gibson, L. J. (1995). The effects of non-periodic microstructure on the elastic properties of two-dimensional cellular solids. International Journal of Mechanical Sciences, 37(11), 1161-1177.

Virk, K., Monti, A., Trehard, T., Marsh, M., Hazra, K., Boba, K., ... \& Farrow, I. R. (2013). SILICOMB PEEK Kirigami cellular structures: mechanical response and energy dissipation through zero and negative stiffness. Smart Materials and Structures, 22(8), 084014.

Zhu, H. X., Knott, J. F., \& Mills, N. J. (1997). Analysis of the elastic properties of open-cell foams with tetrakaidecahedral cells. Journal of the Mechanics and Physics of Solids, 45(3), 319-343.

Zhu, H. X., Hobdell, J. R., \& Windle, A. H. (2001). Effects of cell irregularity on the elastic properties of 2D Voronoi honeycombs. Journal of the Mechanics and Physics of Solids, 49(4), 857-870.

Zhu, H. X., Thorpe, S. M., \& Windle, A. H. (2006). The effect of cell irregularity on the high strain compression of 2D Voronoi honeycombs. International Journal of Solids and Structures, 43(5), 1061-1078. 